\title{
Interoperabilidade e Integração de Sistemas e Dados para Apoio à Tomada de Decisão pela Gestão da Prefeitura de Volta Redonda-RJ: Perspectivas e Desafios
}

\author{
Matheus M. Cruz ${ }^{1}$, Marcos V. A. Delgado ${ }^{2}$, Flavia Bernardini ${ }^{1}$, Vanessa T. Nunes ${ }^{3}$, \\ Carlos A. M. Bastos ${ }^{4}$ \\ ${ }^{1}$ Instituto de Computação - Universidade Federal Fluminense (UFF) \\ Niterói - RJ - Brasil \\ ${ }^{2}$ Instituto de Ciências Humanas e Sociais - Universidade Federal Fluminense (UFF) \\ Volta Redonda - RJ - Brasil \\ ${ }^{3} \mathrm{SE7Ti}$ - Tecnologia e Inovação - Rio de Janeiro - RJ - Brasil \\ ${ }^{4}$ Escola de Engenharia da Universidade Federal Fluminense (UFF) \\ Niterói - RJ - Brasil \\ mmcruzeid.uff.br, marcos.delgado@voltaredonda.rj.gov.br, \\ fcbernardinidic.uff.br, vanunesegmail.com, camalcherbastosdid.uff.br
}

\begin{abstract}
In this Organizational Report, we present the current scenario of the information technology systems and databases of the Municipality of Volta Redonda. We also present the ICT challenges experienced by this municipality, and the ways proposed to face them. We discuss actions to be taken by the Municipality to integrate and interoperate systems and databases to benefit citizens, as we believe in their (re) positioning as a central element of service provision. In addition, in our discussion, we understand that it is of great importance that municipal public managers have integrated and consistent information to assist in the decision-making process.
\end{abstract}

Resumo. Neste Relato Organizacional, apresentamos o cenário atual dos sistemas de tecnologia da informação e bases de dados da Prefeitura Municipal de Volta Redonda. Apresentamos também os desafios de TIC vivenciados por essa municipalidade e os caminhos propostos para enfrentá-los. São discutidas ações que podem ser adotadas pelo Município para integrar e interoperar os sistemas e as bases de dados para beneficiar os cidadãos, pois acreditamos no seu (re)posicionamento como elemento central da prestação do serviço. Além disso, na nossa discussão, entendemos que é de grande importância que os gestores públicos municipais tenham informações integradas e consistentes para auxiliar no processo de tomada de decisão.

\section{Introdução}

A separação das ações do governo por áreas (Educação, Segurança, Saúde, Mobilidade, etc.) segue uma linha conservadora da administração, em que se gerencia por segmentação [SANTOS, 2008]. Este modelo permite um foco mais acurado nos 
problemas de cada setor, tendo sido adotado por muitos Municípios. A informatização da Administração Pública também aconteceu de forma análoga, gerando sistemas independentes, verticalizados, não havendo preocupação com as possíveis interações entre as diversas áreas. Os sistemas foram desenvolvidos desacoplados, seja para a execução das atividades-fim de cada Secretaria, seja para a execução de atividades meio, como gestão documental, compras, execução orçamentária, controle de patrimônio e etc.

No entanto, com as mudanças socioeconômicas que vem transformando o mundo, já não é mais aceitável o isolamento das informações, ou seja, o trabalho estanque, centrado apenas no próprio enfoque, característico das organizações públicas tradicionais [CASTELLS, 1999]. Há a necessidade dos Governos mudarem o foco dos seus serviços para os cidadãos e suas necessidades, o que implica em um nível maior de integração dos dados [LALLANA, 2007; HOPKIRK, 2008] . Por exemplo, os serviços ofertados pelos Municípios estão pulverizados entre as diversas Secretarias, o que dificulta o acesso a eles. A administração municipal precisa integrar essas estruturas fragmentadas, para que os serviços públicos sejam estruturados a partir das necessidades dos cidadãos, conforme vem sendo preconizado por diversos autores no contexto de Cidades Inteligentes [BERNARDINI et al, 2017]. O objetivo é que o cidadão passe a conversar com o Município. Antes, ele falava com as várias Secretarias, sendo obrigado a entender e lidar com a complexidade de cada uma delas. Com a integração e interoperabilidade dessas Secretarias, parte dessa complexidade passa ao próprio Município. O Município de Volta Redonda, localizado no sul fluminense, vem trabalhando justamente na interoperabilidade e integração dos seus diversos sistemas de informação. Cada secretaria possui sistemas e bases de dados isoladas. Tais bases não são integradas, e ainda há problemas para relacioná-las, pois não há um identificador único (como o CPF Cadastro de Pessoa Física) para as mesmas entidades representadas em todas as bases.

O objetivo desse Relato da Organização é discutir o cenário atual de sistemas de software e sistemas de gerenciamento de bases de dados existentes no governo local (secretarias e órgãos da prefeitura) de Volta Redonda, os desafios para solução dos problemas no cenário atual, e as soluções concebidas para tais problemas. Para isso, inicialmente apresentamos na Seção 2 o cenário atual do município, do ponto de vista de soluções computacionais do governo local. Nessa seção, é realizada uma descrição do funcionamento de uma empresa pública municipal responsável pela TIC do Município, a Empresa de Processamento Eletrônico de Dados de Volta Redonda (EPDVR). Ainda, são apresentadas as principais tecnologias empregadas e os desafios atuais enfrentados pelos gestores devido a não integração dos sistemas e bases de dados. Na Seção 3, são discutidos os desafios e as estratégias até então utilizadas por Volta Redonda na interoperabilidade e integração dos seus sistemas computacionais e bases de dados, e quais os impactos trazidos aos processos municipais de governo eletrônicos. Além disso, também são apresentadas as soluções para Volta Redonda minimizar o problema da não integração dos sistemas. Por fim, na Seção 4 são apresentadas conclusões sobre este relato organizacional.

\section{Cenário Atual}

\subsection{Empresa de Processamento Eletrônico de Dados de Volta Redonda - EPDVR}

O município de Volta Redonda apresenta uma característica que o diferencia positivamente dos demais: a existência da EPDVR, uma empresa pública municipal de Tecnologia da Informação e Comunicação (TIC) responsável por gerir e coordenar todas 
as ações estratégicas que envolvam informática, tecnologia e inovação. No Estado do Rio de Janeiro, apenas a capital possui também uma empresa pública municipal de TIC. Por possuir a natureza jurídica de empresa pública, ela visa o lucro, podendo comercializar seus produtos e serviços a outros municípios e empresas privadas. Dessa forma, a EPDVR além de prestar serviços à Prefeitura de Volta Redonda pode ser uma fonte de recursos financeiros ao município.

A empresa ${ }^{1}$ possui mais de 36 anos de parceria com a Prefeitura, o que vem trazendo frutos para ambos, e principalmente, para a população volta-redondense que se beneficia das soluções de tecnologia que foram e estão sendo implantadas na cidade. Podemos citar, como exemplo: o aplicativo FiscalizaVR, para solicitar e acompanhar pedidos de serviços à Prefeitura; o projeto Aldeia Digital, que fornece internet sem fio (wi-fi) gratuita em diversos bairros da cidade; a ferramenta OP Digital, para apoiar o orçamento participativo; a cerca inteligente, que monitora todos os veículos que entram e saem da cidade; o Alvará Fácil, que possibilita a emissão do alvará em até 2 dias; e o Portal dos Exames que disponibiliza os resultados de exames laboratoriais pela internet.

\subsection{Tecnologias}

A Prefeitura Municipal de Volta Redonda faz uso de soluções de TIC há mais de 3 décadas, desde os antigos computadores mainframe. Por esse motivo, possui um legado de tecnologias muito volumoso, heterogêneo e dispare. Em termos quantitativos, existem, na presente data, mais de 78 sistemas ativos e em funcionamento no datacenter da Prefeitura que é administrado pela EPDVR. São sistemas para 10 áreas. Dentre estes, os 3 principais são: sistema tributário, sistema educacional e sistema de saúde. Desse total, 63 deles foram desenvolvidos pela EPDVR. Essas características dificultam a manutenção e gerência dos dados.

A tecnologia utilizada no desenvolvimento desses sistemas é heterogênea, sendo utilizadas tanto linguagens mais antigas como Cobol, Pascal, Delphi, Visual Basic quanto linguagens mais recentes como Php, Java e Python. Os sistemas gerenciadores de bancos de dados (SGBD) também são vários, tais como OpenBASE, FireBird, MySQL, SQL Server, PostgreSQL, Oracle e Microsoft Access. Essas características aumentam a necessidade de metodologias e tecnologias para garantir a interoperabilidade entre os sistemas, já que essa complexidade tende a aumentar.

\subsection{Desafios Enfrentados}

Em virtude da grande diversidade de sistemas e tecnologias que foram sendo incorporadas ao portfólio da Prefeitura, torna-se complexo o processo de integração e interoperabilidade entre as soluções [ALBUQUERQUE FILHO, 2010]. Um dos fatores dessa complexidade é a escassez de recursos humanos especializados em tecnologias não mais adotadas, utilizadas em sistemas legados que ainda estão em funcionamento. A ausência de documentação dos sistemas e metadados não padronizados também contribuem para a complexidade do processo. Outro fator que também prejudica essa integração é o custo elevado para a implementação das adequações necessárias. Além disso, a maioria das secretarias possuem sistemas de informação isolados e estanques, que não se comunicam com os sistemas de outras secretarias. Esse fator dificulta a

\footnotetext{
${ }^{1}$ Informações complementares da EPDVR e dos projetos em parceria com a Prefeitura Municipal de Volta Redonda estão disponíveis em: http://www.epdvr.com.br
} 
integração de informações para auxiliar aos gestores nas tomadas de decisões. Para citar alguns exemplos, que já ocorreram, atualmente não se consegue gerar os relatórios a seguir de forma automatizadas: (i) relação de alunos matriculados na rede municipal de educação que foram atendidos na rede municipal de saúde; (ii) os servidores municipais que possuem filhos matriculados na rede municipal de educação; e (iii) cidadãos que recebem benefícios assistenciais e que possuem algum imóvel próprio registrado no município.

Para atender a essas e outras situações para auxiliar os gestores nas tomadas de decisões, os relacionamentos são realizados manualmente e diretamente nas bases de dados, desde que seja possível comparar informações equivalentes. Assim, a falta de integração dos sistemas interfere também no dia a dia dos cidadãos, que constantemente tem o retrabalho de fornecer as mesmas informações e documentos sempre que buscam serviços prestados pelo Município. Por exemplo, quando uma mãe matricula seus filhos nas escolas municipais precisará preencher um formulário online com todos os dados e quando for buscar atendimento em alguma unidade de saúde precisará também fornecer dados semelhantes que poderiam ser reutilizados daqueles informados na escola.

Volta Redonda iniciou esse processo de integração em alguns cenários. O FiscalizaVR é um aplicativo único pelo qual o cidadão pode solicitar serviços ou informações à todas as Secretarias. Pelo aplicativo é possível, por exemplo, solicitar a poda de uma árvore ou a troca da lâmpada de um poste, ou tapar buraco de uma rua ou solicitar visita técnica da defesa civil, dentre outros. Outro exemplo de integração está relacionada ao uso do Módulo Financeiro do software público E-Cidade, adotado pela Prefeitura. Todos os órgãos e entidades da Administração Pública Municipal utilizam esse módulo do software, o que torna mais eficiente a consolidação da prestação de contas anual do Município, pois os órgãos e entidades municipais utilizam os mesmos planos de contas e rubricas orçamentárias em um banco de dados único. No entanto, é importante observar que mecanismos para promover a interoperabilidade operacional ainda são necessários, bem como mudanças culturais organizacionais para melhorar a interoperabilidade entre os diversos setores.

\section{Soluções Propostas para Volta Redonda}

O desafio do Município em construir uma gestão mais eficiente faz com que o uso das Tecnologias da Informação e da Comunicação (TICs) e, principalmente, a integração e a interoperabilidade entre sistemas estejam no centro do processo de melhoria.

\subsection{Interoperabilidade entre Sistemas e Bases de Dados}

A aplicação da interoperabilidade no contexto municipal tem como principal objetivo garantir a interação de sistemas e órgãos de maneira eficaz e eficiente [CUNHA, et al., 2005]. Os conceitos de interoperabilidade e integração são muitas vezes utilizados como sinônimos na área de TIC [MESQUITA, 2010]. Entretanto, esses conceitos são distintos, embora complementares, segundo o Guia de Interoperabilidade do Governo Federal [GOVERNO FEDERAL, 2012]. "Integração refere-se ao processo de conectar dois ou mais sistemas gerando uma dependência tecnológica entre eles. Interoperabilidade refere-se ao processo de comunicação de dois ou mais sistemas sem a geração de uma dependência tecnológica entre eles". Em muitos casos, a integração entre sistemas é a melhor solução [SANTOS, 2008]. 
A LAI (Lei de Acesso à Informação), sancionada em 18 de novembro de 2011, reforça o uso da interoperabilidade na busca pela publicidade dos dados. Ao garantir, virtualmente, um maior acesso aos direitos do cidadão e aumentar a sua parcela de participação na gestão do Município, a interoperabilidade possibilita a expansão do exercício da cidadania. Isso permite, ao menos tecnicamente, o acesso, pelo cidadão, a serviços e informações atualmente pulverizados e inacessíveis. Uma maneira de atingir esse objetivo é a publicação de dados abertos em portais na web, em que a sociedade pode acessar e reutilizar dados brutos para fins diversos [STI, 2012].

Dessa forma, com o objetivo de ampliar essa disponibilização dos dados, a Prefeitura pretende primeiramente incorporar padrões definidos pelo e-PING aos processos de desenvolvimento de softwares da EPDVR, que possibilitam a troca de informações entre as diversas esferas de governo (municipais, estaduais e federais). Além disso, a Prefeitura pretende identificar informações que possam ser compartilhadas pelas diversas Secretarias, tais como documentos comprobatórios de regularidade de situação do cidadão, atestados e certidões, em uma base de dados oficial para toda a administração pública municipal, de modo que os órgãos e entidades possam obtê-los diretamente sem a necessidade do cidadão ter que pedir a outro órgão municipal. A Prefeitura pretende também permitir a realização de buscas a recursos informacionais heterogêneos, armazenados em diferentes servidores na rede, utilizando- se de uma interface única sem o cidadão precisar tomar conhecimento de onde nem como estes recursos estão armazenados. Essas ações propiciarão ganhos imediatos como: melhor uso dos recursos tecnológicos, menor tempo de resposta às necessidades de criação e manutenção de novas soluções tecnológicas e provimento de serviços aos cidadãos com mais clareza e qualidade.

As ações de interoperabilidade propostas têm o potencial de gerar instrumentos para os gestores para uma melhor tomada de decisão, uma melhor coordenação dos programas e serviços de governo e redução de custos, por meio da visão integrada dos processos do governo e do reuso de soluções tecnológicas, como pode ser observado na Figura 1. Uma maneira de resolver a questão de integração é implantar outros módulos, descrito na seção a seguir.

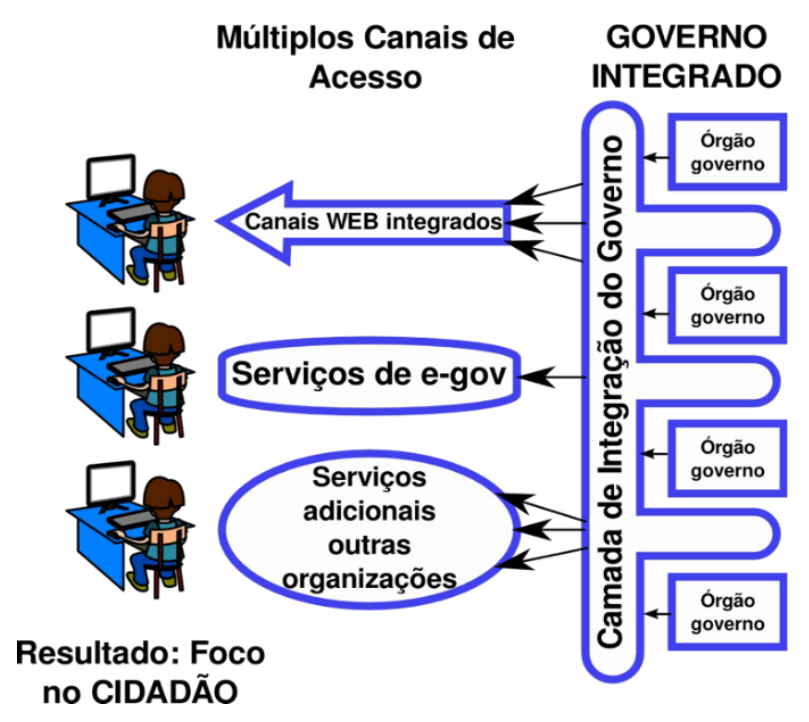

Figura 1. Visão integrada dos processos do governo 


\subsection{Implantação de Demais Módulos do Sistema E-Cidade}

Instituições públicas como Prefeituras, por exemplo, utilizam cada vez mais os softwares de gestão para cuidar da administração de seus recursos [ALBUQUERQUE, 2010]. Esses softwares, por sua vez, estão em constante evolução e hoje podem ser divididos em três categorias: isolado, integrado e ERP. Os softwares isolados atendem cada setor da organização de maneira individual, dificultando a transferência eletrônica de dados para outros setores do mesmo órgão. Com sistemas integrados, é possível transferir informações entre setores, através de rotinas de exportação e importação. No entanto, cada área da organização é responsável pela manutenção do seu conjunto de informações, o que gera o risco de desatualização e a duplicação de informações e tabelas de dados. Por fim, os ERPs são mais aconselháveis para a gestão eficiente das instituições

O e-CIDADE é um sistema que se enquadra na categoria ERP. Nesta categoria, o software é livre para prefeituras, e atende à instituição (prefeitura de Volta Redonda) como um todo. Ele processa as informações de todos os setores de forma mais eficiente, não permitindo a existência de "ilhas" de informação ou duplicidade de tabelas de dados e garantindo a sincronia entre as áreas administrativas. Isso é devido à engenharia funcional do e-CIDADE, que melhora o fluxo de informações dentro da organização, fazendo com que os dados estejam sempre atualizados, diminuindo gastos em manutenção de integração de sistemas. No futuro, o avanço tecnológico e a popularização de plataformas como o e-CIDADE possibilitarão que as pessoas acessem informações da Prefeitura usando apenas o celular por meio de um código de acesso único, para todas as suas informações pessoais e visualizar os dados de diferentes setores, como tributário, social, saúde, educação e saneamento, por exemplo. Dessa forma, será possível que o cidadão consiga acessar as notas dos seus filhos que estudam na rede municipal de educação e as contas de água da sua residência, por exemplo.

Para que esse cenário se torne realidade, um dos caminhos envolve o planejamento e investimento em infraestrutura tecnológica e plataformas eficientes similares ao eCIDADE, que possui uma base de dados única para os diversos sistemas de informação conforme Figura 2. Esse caminho permite que sistemas computacionais sejam capazes de atender, simultaneamente, às diversas áreas da Administração Municipal (saúde, educação, saneamento, infraestrutura pública, etc), que pode levar a prefeitura a atingir o cenário almejado. O primeiro módulo do e-CIDADE implantado em toda a Administração Municipal foi o Financeiro. Ocorreu em meados de 2014, para atender ao Plano de Contas Aplicado ao Setor Público (PCASP) e à uniformização do plano de contas de todo o Município. Como resultado dessa integração, a Administração obteve de imediato a consolidação automática das informações contábeis e financeiras, pois só um sistema único o faria com precisão e sem a intervenção manual. $\mathrm{O}$ ganho com esta integração das informações proporcionou maior consistência dos dados que eram gerados e entregues ao TCE RJ, SICONFI e outras entidades governamentais. Antes dessa implantação, a Administração consolidava manualmente todas as contas que eram geradas pelos diversos sistemas utilizados pelas instituições municipais, que gerava ineficiência, erros e desperdício de recursos ao Município. Os próximos módulos a serem implantados serão o patrimonial (patrimônio, almoxarifado, compras, licitação e contratos) e de Recursos Humanos (pessoal, folha de pagamento e estágio probatório) em toda a Administração Pública Municipal. 


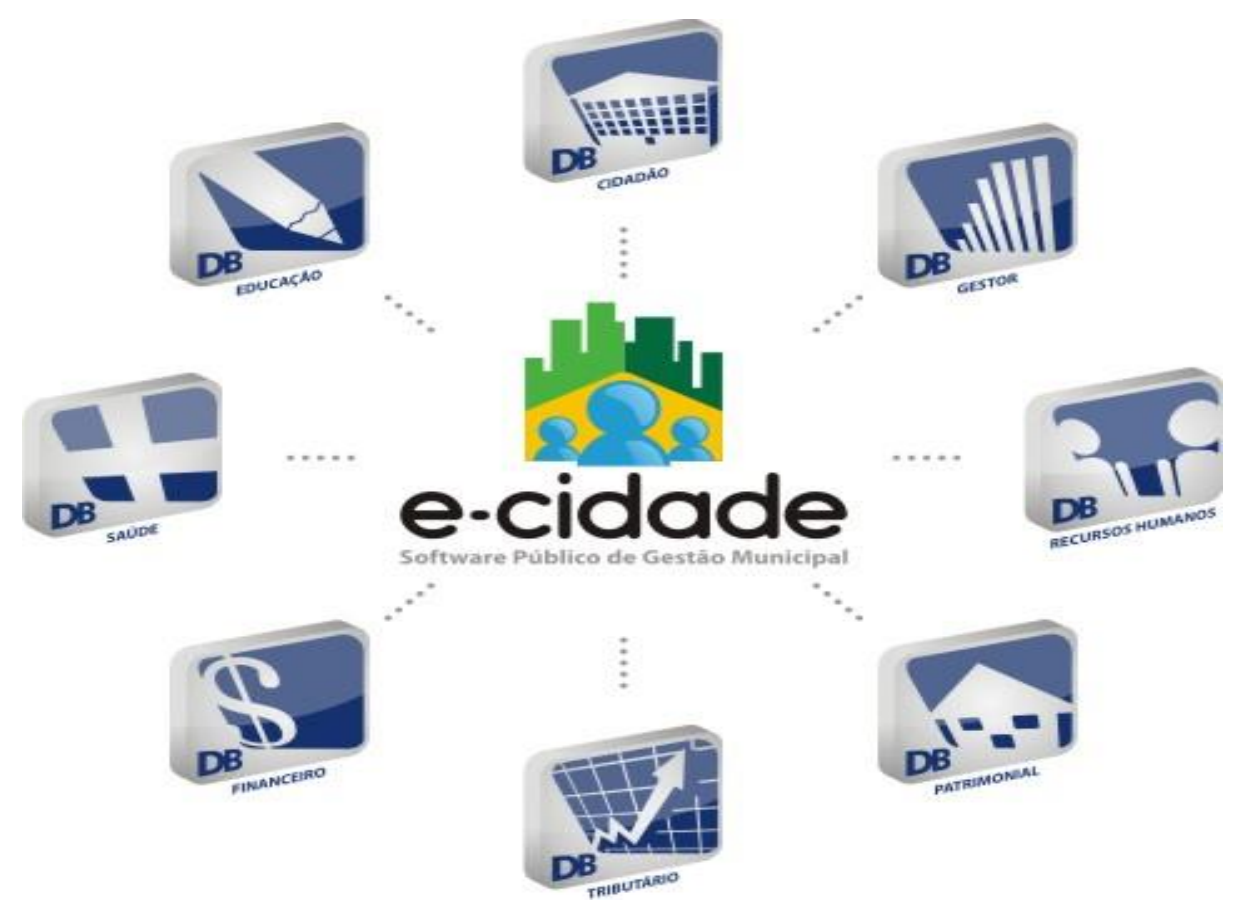

Figura 2. Módulos do Sistema E-Cidade

\section{Conclusões}

Neste Relato Organizacional, discutimos a importância e os benefícios da integração e interoperabilidade dos sistemas de tecnologia da informação e bases de dados dos órgãos e entes da Prefeitura de Volta Redonda. A interoperabilidade possibilita a construção de portais de dados abertos, o que pode levar à expansão do exercício da cidadania, permitindo o acesso pelo cidadão a serviços e informações atualmente pulverizados e de difícil acesso [ALBUQUERQUE FILHO, 2010]. Os gestores municipais também são beneficiados com as ações propostas para Volta Redonda pois terão acesso a informações integradas, consistentes e em tempo real, imprescindíveis para tomada de decisões com maior efetividade e eficiência.

No entanto, é importante destacar que as soluções construídas para integração de maneira organizacional e a implementação dos outros módulos do e-Cidade não resolve todos os problemas de integração e interoperabilidade em todos os cenários de governo local. Isso é devido a ter muitos sistemas existentes, inclusive sistemas legados, que não serão facilmente substituídos. Além disso, é importante considerar a evolução desses ecossistemas de sistemas computacionais e bases de dados. Nesse sentido, novas metodologias e tecnologias para garantir uma evolução sustentável desse ecossistema é importante nesse contexto.

\section{Referências Bibliográficas}

ALBUQUERQUE FILHO, A. C. D. P.; PESSI, P. Modelo de Interoperabilidade para Municípios do Estado de São Paulo: integrando sistemas com compartilhamento, reúso 
e itercâmbio de informações. Primeira Edição. ed. São Paulo: Fundação Prefeito Faria Lima - CEPAM, 2010. 76 p.

BERNARDINI, F.; VITERBO, J.; VIANNA, D.S .; MARTINS, C.B.; MEDEIROS, A.P.; MEZA, E.B.M.; MORATORI, P.B.; BASTOS, C.A.M. General Features of Smart City Approaches from Information Systems Perspective and Its Challenges. Chapter 3. In: BOSCARIOLI, C.; ARAUJO, R.; MACIEL, R. (orgs). I GranDSI-BR - Grand Research Challenges in IS in Brazil - 2016-2026, 2017.

CASTELLS, M. A sociedade em rede, vol. 1. São Paulo: Paz e Terra, 1999.

COMITÊ EXECUTIVO DE GOVERNO ELETRÔNICO. E-Ping, Padrões de Interoperabilidade de Governo Eletrônico. Versão 4.0. ed. Brasília: Governo do Brasil, 2012.

CUNHA, M. X. C. D.; SOUZA JUNIOR, M. F. D.; ALMEIDA, H. O. D. Dificuldades com integração e interoperabilidade de sistemas de informação nas instituições públicas de ensino - um estudo de caso no CEFET-AL. anais do XII SIMPEP. Bauru - SP: [s.n.]. 2005. p. 11.

GOVERNO FEDERAL. Padrões de Interoperabilidade de Governo Eletrônico. Guia de Interoperabilidade: Manual do Gestor Ministério do Planejamento, Orçamento e Gestão, 2012. Disponivel em: <https://www.gov.br/governodigital/pt-br/governancade-dados/Guia de Interoperabilidade_Manual_do_Gestor_2012.pdf>. Acesso em: 11 Maio 2020.

HOPKIRK, A. Case Study: The e-Government Interoperability Framework (e-GIF), procurement and innovation policies in action. London: The National Computing Center, 2008.

LALLANA, D. E. C. E-Government Interoperability: a review of Government Interoperability Frameworks in selected countries. 1a Edição. ed. Bangkok: United Nations Development Programme, 2007.

MESQUITA, C. D. S. F.; BRETAS, N. L. (Org.) Panorama da interoperabilidade no Brasil. 1. ed. Brasília: MP/SLTI, 2010. 251 p. ISBN ISBN 978-85-89199-07-0.

SANTOS, E. M. D. Desenvolvimento e implementação de padrões de interoperabilidade em governo eletrônico no Brasil. Universidade de São Paulo. São Paulo, p. 184. 2008. (CDD 658.4038).

SECRETARIA DE TECNOLOGIA DA INFORMAÇÃO (STI), MINISTÉRIO DO PLANEJAMENTO ORÇAMENTO E GESTÃO (MPOG). "Cartilha Técnica para Publicação de Dados Abertos no Brasil v1.0", 2012. Disponível em: http://www.dados.gov.br/pagina/cartilha-publicacao-dados-abertos : Acessado em: 13 Maio 2017. 